\title{
Clinical efficacy evaluation and prevention of adverse reactions in a randomized trial of a combination of three drugs in the treatment of cancerous pudendal neuralgia
}

\author{
Junjie Hou ${ }^{1,2}$, Yifan $\mathrm{Lin}^{2}$, Yanqiu Fang ${ }^{2}$, Xiaonan $\mathrm{Li}^{2}$, Xiao-Nan $\mathrm{Li}^{2}$, Ying Yang ${ }^{2}$, Ning Liu ${ }^{3}$, Xianzhuo Jiang ${ }^{2}$, \\ Yingying $\mathrm{Yu}^{2}$, Ying Zhou ${ }^{2}$, Xuguang $\mathrm{Mi}^{2}$, Zhiqiang $\mathrm{Ni}^{2}$, Xiaodan $\mathrm{Lu}^{2}$, Ning-Yi Jin ${ }^{1,4}$ \\ ${ }^{1}$ Medical College, Yanbian University, Yanji, China; ${ }^{2}$ Department of Comprehensive Oncology, Jilin Province People's Hospital, Changchun, China; \\ ${ }^{3}$ General Surgery of The First Clinical Hospital of Jilin Academy of Chinese Medicine Sciences, Changchun, China; ${ }^{4}$ Laboratory of Molecular \\ Virology and Immunology, Institute of Military Veterinary Medicine, Academy of Military Medical Science, Changchun, China \\ Contributions: (I) Conception and design: J Hou, Y Fang, Xi Li; (II) Administrative support: None; (III) Provision of study materials or patients: Fy \\ Lin, X Jiang, Y Yu; (IV) Collection and assembly of data: N Liu, Y Zhou, Z Ni; (V) Data analysis and interpretation: Y Yang, X Mi, X Lu, NY Jin; (VI) \\ Manuscript writing: All authors; (VII) Final approval of manuscript: All authors. \\ Correspondence to: Ning-Yi Jin. Medical College, Yanbian University, Yanji 133002, China; Laboratory of Molecular Virology and Immunology, \\ Institute of Military Veterinary Medicine, Academy of Military Medical Science, Changchun 130122, China. Email: ningyik1@126.com.
}

Backgroundk To explore the clinical efficacy, safety, and prevention of major adverse reactions of the nonsteroidal anti-inflammatory drug celecoxib combined with OxyContin and Pregabalin in the treatment of cancerous pudendal neuralgia.

Methods: A total of 51 patients presenting with pelvic malignancies with cancerous pudendal neuralgia were selected, and random number table method was used to allocate them to either the experimental group $(n=27)$ or control group $(n=24)$. The control group was treated with OxyContin combined with Pregabalin, and the experimental group was treated with Celecoxib on the basis of the control group.

Results: At 24 hours after treatment, the clinical effective rate of the experimental group was $92.6 \%$, which was significantly higher than the $66.7 \%$ of the control group $(\mathrm{P}<0.05)$. The numerical rating scale (NRS) scores of the 2 groups of participants on the 7th and 14th days after treatment were lower than before treatment $(\mathrm{P}<0.05)$, and the NRS scores of the participants in the experimental group had decreased more significantly. At the same time, the average daily consumption of OxyContin on the 7th and 14th day of the experimental group was lower than that of the control group $(\mathrm{P}<0.05)$. Compared with the control group, the incidence of constipation and dysuria in the experimental group was significantly reduced $(\mathrm{P}<0.05)$. Co-occurring in both groups during treatment, 10 participants with urinary dysfunction were treated with tamsulosin hydrochloride sustained-release capsules, no urinary retention occurred, catheterization was avoided, tamsulosin hydrochloride sustained-release capsules could be stopped after 1 week, and urination was smooth $(\mathrm{P}<0.05)$. After treatment, the quality of life of the 2 groups of participants had improved compared to before treatment, and the improvement was more significant in the experimental group.

Conclusions: When treating patients with cancerous pudendal neuralgia with OxyContin and Pregabalin, the addition of celecoxib has a significant effect, which can effectively improve the patient's pain, improve their quality of life to a certain extent, and reduce the consumption of OxyContin. Lowering the dose of OxyContin reduces the occurrence of adverse reactions related to the drug, especially the incidence of constipation and urinary retention. Tamsulosin hydrochloride sustained-release capsules can effectively relieve urinary disorders caused by OxyContin.

Trial registration: Chinese Clinical Trial Registry ChiCTR2100046045.

Keywords: Celecoxib; OxyContin; Pregabalin; cancerous pudendal neuralgia 
Submitted Feb 03, 2021. Accepted for publication Apr 23, 2021.

doi: 10.21037/apm-21-590

View this article at: http://dx.doi.org/10.21037/apm-21-590

\section{Introduction}

Cancer pain accompanies $76 \%$ of advanced tumors, and the proportion of cancer pain is even higher in patients who are dying $(1,2)$. More than $40 \%$ of dying patients have neuropathic pain, which affects their daily activities (3). Pudendal neuralgia is a kind of typical neuropathic pain and is characterized by acupuncture-like, electric shocklike, or burning-like pain that occurs in the area innervated by the pudendal nerve (4). This neuropathic pain is often complex, especially when the pelvic and abdominal cavity are infiltrated by cancers often associated with pudendal neuralgia, such as cervical, bladder, rectal, prostate, ovarian, and endometrial cancer. The direct infiltration and compression of these tumors and the inflammatory factors they release often invade the pudendal nerve plexus, leading to the occurrence of pudendal neuralgia and inflicting longterm mental and physical torture on patients. Pregabalin is a gamma-aminobutyric acid (GABA) analog with anti-anxiety, anticonvulsant, and analgesic properties. It has been widely used in the treatment of neuropathic pain $(5,6)$, including that of cancer. Opioids are the cornerstone of cancer pain treatment (7), and OxyContin is the representative opioid. Anticonvulsants (Pregabalin) and OxyContin are often used clinically to treat cancerous neuropathic pain. OxyContin is a strong opioid analgesic and has no a "ceiling effect" in increasing doses, but adverse reactions can increase and become aggravated with increasing doses, manifesting as dizziness, nausea, constipation, and dysuria caused by the drug persistence. Serious complications can arise from these adverse reactions, including intestinal obstruction and urinary retention. This leads to a bottleneck in the treatment of cancer pain (8), which limits the incremental clinical application of opioids (9), especially in pudendal neuralgia patients with pelvic and abdominal tumors, because these patients already have a certain degree of urinary and defecatory disorder before treatment $(10,11)$. Celecoxib is a non-steroidal anti-inflammatory drug, a specific cyclooxygenase-2 (COX-2) inhibitor. Studies have shown that celecoxib combined with opioids for the treatment of cancer pain can not only improve the symptoms of cancer pain, but also reduce the application dose of opioids and reduce the occurrence of adverse reactions (12). Previous studies on celecoxib in pubic neuralgia mainly focused on the efficacy of benign diseases caused by pubic neuralgia, mostly with single drug or two-drug combination. The study was based on opioids in combination with celecoxib and pregabalin in patients with advanced cancer. This study is the first comprehensive treatment of cancer pain based on the complex mechanism, and the efficacy and adverse reactions were observed. In addition, this study is the first to observe and manage adverse events in a trial, demonstrating that a three-drug combination can increase efficacy and downregulate opioid doses and adverse reactions associated with the drug. This study aimed to observe the effectiveness and safety of the combination of celecoxib, OxyContin, and Pregabalin in the treatment of cancerous pudendal neuralgia, and at the same time apply tamsulosin hydrochloride to patients with difficult urinary dysfunction symptoms during treatment. We present the following article in accordance with the CONSORT reporting checklist (available at http://dx.doi. org/10.21037/apm-21-590).

\section{Methods}

\section{Research participants}

The inclusion criteria for this study were as follows: (I) patients diagnosed as having malignant tumors with advanced tumors of pudendal neuralgia; (II) no history of opioid abuse before admission; (III) no history of mental illness, capable of verbal expression, can accurately express pain; (IV) estimated survival time greater than 4 weeks. The exclusion criteria were as follows: (I) patients with nausea and vomiting, indwelling catheterization, and intestinal obstruction before admission; (II) pregnant and lactating women; (III) those allergic to the drugs used in this study; (IV) severe gastrointestinal diseases and cardiac insufficiency. A total of 51 patients with malignant tumors with cancerous pudendal neuralgia were recruited from June 2019 to June 2020 in Jilin Province People's Hospital. All procedures performed in this study involving human participants were in accordance with the Declaration of Helsinki (as revised in 2013). This study was approved by Jilin Province People's Hospital ethics committee. All participants provided informed consent and signed an informed consent. Using a random number table method, participants were allocated 
Table 1 Baseline data of the patient

\begin{tabular}{|c|c|c|c|}
\hline Variable & The experimental group $(n=27)$ & Control group $(n=24)$ & $\mathrm{P}$ \\
\hline Male & 12 & 13 & 0.579 \\
\hline Female & 15 & 11 & \\
\hline Age & $57.22 \pm 12.38$ & $58.62 \pm 14.36$ & 0.710 \\
\hline Carcinoma of the rectum & 9 & 8 & $>0.999$ \\
\hline Cervical cancer & 6 & 4 & 0.732 \\
\hline Bladder cancer & 5 & 6 & 0.736 \\
\hline Prostatic cancer & 6 & 5 & $>0.999$ \\
\hline
\end{tabular}

to either the experimental group $(n=27)$ or the control group $(n=24)$. The experimental group included 12 males and 15 females, with an average age of $57.22 \pm 12.38$ years. The disease types in the experimental group included 9 cases of rectal cancer, 6 cases of cervical cancer, 5 cases of bladder cancer, 6 cases of prostate cancer, and 1 case of ovarian cancer. The control group included 13 males and 11 females, with an average age of $58.62 \pm 14.36$ years. The types of diseases in the control group included 8 cases of rectal cancer, 4 cases of cervical cancer, 6 cases of bladder cancer, 5 cases of prostate cancer, and 1 case of uterine endometrial cancer. There was no statistically significant difference in gender, age, and disease type between the 2 participant groups $(\mathrm{P}>0.05)$, and they were comparable (Table 1$)$.

\section{Treatment methods}

In the control group, pain titration was performed with immediate-release morphine hydrochloride tablets/ injection. After the titration, the total dose of the 24-hour immediate-release morphine drug was calculated and converted into equivalent OxyContin (Mengdi Pharmaceutical Co., Ltd., Beijing, China). The dose was taken orally every 12 hours, combined with $75 \mathrm{mg}$ of Pregabalin (Chongqing Saiwei Pharmaceutical Co., Ltd., Chongqing, China), orally twice a day. In the experimental group, $200 \mathrm{mg}$ of celecoxib (Pfizer Pharmaceuticals LLC, NY, USA) was added on the basis of the control group, orally every 12 hours. Both groups were administered the same dose of laxative drugs to prevent constipation. Symptomatic drug treatment was provided when drug- related adverse reactions occured during treatment, and tamsulosin hydrochloride sustained-release capsules were given to patients with urination disorders (Astellas Pharmaceuticals Co., Ltd., Beijing, China) at a dose of $0.2 \mathrm{mg}$, orally once a day.

\section{Observation indicators}

The following indicators were recorded separately: (I) the numerical rating scale (NRS) scores of the 2 groups of patients before and after treatment $(0-10$ points, 0 is the most satisfactory, 10 points are poor); (II) the dose of OxyContin applied to the 2 groups of participants; (III) the occurrence of adverse reactions after the drug, including dizziness, nausea and vomiting, constipation, dysuria, lethargy, peripheral edema, and so on. The efficacy evaluation criteria were as follows: effective, the pain basically disappeared or completely disappeared; ineffective, the pain did not significantly improve or even worsened.

\section{Statistical analysis}

The software SPSS 20.0 (IBM, Armonk, NY, USA) was used to perform $t$-test and chi-squared test on the data, and $\mathrm{P}<0.05$ was considered statistically significant.

\section{Results}

\section{Comparison of efficacy}

The treatment process of the two groups was shown in 


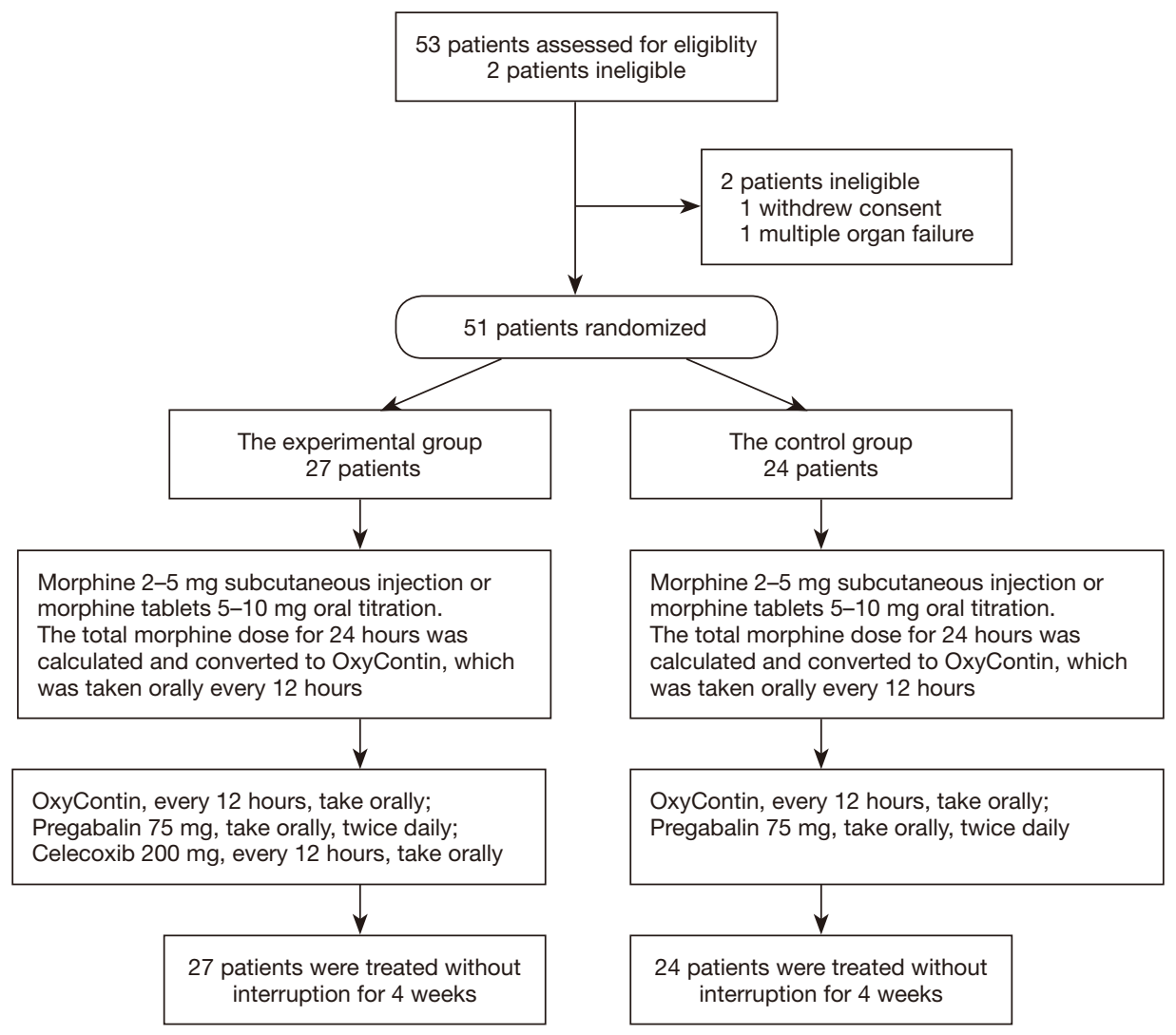

Figure 1 Participant Flow Chart.

Figure 1. At 24 hours after treatment, the clinical effective rate of participants in the experimental group was $92.6 \%$, higher than $66.7 \%$ in the control group, $\chi^{2}=5.312, \mathrm{P}=0.021$, and the difference was statistically significant $(\mathrm{P}<0.05)$ (Table 2).

\section{Comparison of NRS scores after treatment}

The NRS scores of the 2 groups of participants on the 7 th and 14th days after treatment were lower than those before the treatment, the NRS score of the experimental group decreased more significantly, and the differences were statistically significant $(\mathrm{P}<0.05)$ (Table 3).

\section{Comparison of average daily dosage of OxyContin after treatment}

Compared with the control group, the average daily consumption of OxyContin in the experimental group was significantly less on the 7th and 14th days after treatment, and the difference was statistically significant $(\mathrm{P}<0.05)$
(Table 4).

\section{Patient quality of life improved after treatment}

After treatment, the quality of life of both groups of participants had improved compared with that before treatment, the improvement of the experimental group was more significant, and the differences were statistically significant $(\mathrm{P}<0.05)$ (Table 5).

\section{Comparison of adverse reactions}

Compared with the control group, the incidence of constipation and dysuria in the experimental group was significantly reduced, and the difference was statistically significant $(\mathrm{P}<0.05)$. Among them, participants with dysuria were given tamsulosin hydrochloride sustained-release capsules symptomatically. With this treatment, symptoms improved and no urinary retention occurred; however, there was no significant difference in the incidence of nausea, vomiting, lethargy, dizziness, and peripheral edema between 
Table 2 Clinical efficacy of the two groups of patients

\begin{tabular}{lcccc}
\hline Group & Effective number of cases & Effective ratio/\% & Number of invalid cases & Invalid ratio/\% \\
\hline Experimental $(n=27)$ & 25 & 92.6 & 2 & 7.4 \\
Control $(n=24)$ & 16 & 66.7 & 8 & 33.3 \\
\hline
\end{tabular}

Table 3 Comparison of the average daily dosage of OxyContin after treatment in the two groups $(\bar{x} \pm s)$

\begin{tabular}{lccc}
\hline \multirow{2}{*}{ Group } & \multicolumn{3}{c}{ NRS score } \\
\cline { 2 - 4 } & Before treatment & 7th day after treatment & 14th day after treatment \\
\hline Experimental $(\mathrm{n}=27)$ & $6.8 \pm 0.9$ & $2.1 \pm 1.2$ & $1.9 \pm 1.1$ \\
Control $(\mathrm{n}=24)$ & $6.6 \pm 1.1$ & $3.7 \pm 1.3$ & $3.9 \pm 1.1$ \\
$t$ value & 0.714 & 4.570 & 6.762 \\
$\mathrm{P}$ value & 0.472 & $<0.001$ & $<0.001$ \\
\hline
\end{tabular}

NRS, numerical rating scale.

Table 4 Comparison of the average daily dosage of OxyContin after treatment in the two groups $(\bar{x} \pm \mathrm{s})$

\begin{tabular}{lcc}
\hline \multirow{2}{*}{ Group } & \multicolumn{2}{c}{ Average daily dose $(\mathrm{mg})$} \\
\cline { 2 - 3 } & 7th day after treatment & 14th day after treatment \\
\hline Experimental $(\mathrm{n}=27)$ & $42.62 \pm 12.18$ & $45.63 \pm 11.96$ \\
Control $(\mathrm{n}=24)$ & $59.26 \pm 10.87$ & $66.71 \pm 10.18$ \\
$t$ value & 6.161 & 6.309 \\
$\mathrm{P}$ value & $<0.001$ & $<0.001$ \\
\hline
\end{tabular}

Table 5 Comparison of quality of life scores between the two groups $(\bar{x} \pm \mathrm{s})$

\begin{tabular}{|c|c|c|c|c|c|}
\hline Group & Before treatment & $24 \mathrm{~h}$ after treatment & $1 \mathrm{wk}$ after treatment & $2 \mathrm{wk}$ after treatment & 4 wk after treatment \\
\hline Control & $31.43 \pm 6.76$ & $44.35 \pm 7.57^{\triangle}$ & $41.12 \pm 7.10^{\Delta}$ & $37.96 \pm 5.83^{\Delta}$ & $36.19 \pm 7.27^{\triangle}$ \\
\hline$t$ value & 0.405 & 2.399 & 3.229 & 3.755 & 1.762 \\
\hline$P$ value & 0.676 & 0.018 & 0.003 & $<0.001$ & 0.081 \\
\hline
\end{tabular}

" $\triangle$ " means $P<0.05$ vs. before treatment.

the 2 groups $(\mathrm{P}>0.05)$ (Table 6).

Compared with the control group, the liver and kidney function indexes of the experimental participants did not increase significantly 4 weeks after treatment, and the difference was not statistically significant $(\mathrm{P}>0.05)$ (Table 7$)$.

\section{Discussion}

The incidence of malignant tumors is increasing annually, as is the number of patients suffering from cancer pain, which seriously affects the quality of life of cancer patients and their families, especially for those with advanced tumors. How to treat advanced cancer pain through a low-toxic, effective, and non-invasive way has become a common problem faced by clinicians. Neuropathic pain is a common type of cancer pain, and it is also a thorny problem in current treatment. Among patients with cancer pain, at least a third have been diagnosed with neuropathic pain (13). 
Table 6 Comparison of adverse reactions between the two groups

\begin{tabular}{lcccccc}
\hline Group & Nausea and vomiting & Lethargy & Dizziness & Dysuria & Constipation & Peripheral edema \\
\hline Experimental $(\mathrm{n}=27)$ & 8 & 4 & 2 & 2 & 13 & 2 \\
Control $(\mathrm{n}=24)$ & 7 & 3 & 4 & 8 & 20 & 2 \\
$t$ value & 0.001 & 0.000 & 0.347 & 3.898 & 5.230 & 0.000 \\
$\mathrm{P}$ value & 0.971 & 1.000 & 0.556 & 0.048 & 0.022 & 1.000 \\
\hline
\end{tabular}

Table 7 Comparison of liver and kidney function changes between the two groups of patients 4 weeks after treatment $(\bar{x} \pm s)$

\begin{tabular}{lccc}
\hline Group & ALT (IU/L) & AST (IU/L) & $\operatorname{Cr}(\mu \mathrm{mol} / \mathrm{L})$ \\
\hline Control group & $120.54 \pm 73.65$ & $115.30 \pm 68.27$ & $148.82 \pm 94.06$ \\
Test group & $133.27 \pm 77.29$ & $126.24 \pm 75.39$ & $135 \pm 83.34$ \\
$t$ value & -0.920 & -0.883 & 0.846 \\
P value & 0.359 & 0.406 & 0.339 \\
\hline
\end{tabular}

ALT, alanine transaminase; AST, aspartate transaminase; $\mathrm{Cr}$, creatinine.

Pudendal neuralgia is a special type of neuropathic pain, caused by chronic compression of the pudendal nerve and/ or tumor infiltration caused by the clinical manifestations, which often cannot be properly and effectively treated (14). The pudendal nerves have many branches, are densely distributed in the pelvic cavity, and travel in the pudendal nerve tube together with the pudendal blood vessels, which means they often cause severe pain after being compressed and infiltrated by the tumor, and may also be accompanied by bladder, intestinal, or sexual dysfunction. It seriously affects the quality of life of those affected (15), especially when opioids are used to treat cancer pain. Opioids can cause urinary weakness, urinary retention, constipation, and other symptoms, so they often aggravate the bladder and intestines of patients with pudendal neuralgia or symptoms such as sexual dysfunction. Tumors are often associated with inflammation, and inflammation can induce the production of COX-2, which is different from the expression of cyclooxygenase-1 (COX-1) in most cells. It is expressed and significantly increased by tumor cells and stromal cells in an inflammatory state (16). The action of COX-2 can convert arachidonic acid into prostaglandins, leading to the synthesis and accumulation of inflammatory substances, causing edema, local inflammation, and pain (17). More studies have shown that the release of inflammatory factors [such as tumor necrosis factor- $\alpha$ (TNF- $\alpha$ ), interleukin 1 (IL-1), IL-6, and so on] from tumors and surrounding inflammatory cells is closely related to the occurrence of neuropathic pain, and the increase of inflammatory factors is associated with the severity of pain. There is a clear positive correlation between inflammatory cytokines and neuropathic pain (18). Clinically, increasing the dose of opioids or combining with Pregabalin has a certain effect in the treatment of cancerous pudendal neuralgia, but with the increasing analgesic effect, the adverse reactions increase correspondingly, caused by the increased dose of opioids. The incidence of symptoms such as constipation and urinary disturbance has also increased in this way (6). The special anti-inflammatory effect of celecoxib is based on its inhibition of COX-2 activity, which can prevent inflammatory prostaglandin substances. The use of celecoxib has achieved both antiinflammatory and analgesic effects $(19,20)$. Studies have shown that celecoxib and opioids can effectively alleviate the occurrence of cancer pain, without increasing the risk of gastrointestinal and cardiovascular diseases, and can reduce the adverse reactions caused by the increase of opioids to a certain extent (21). As a specific COX-2 inhibitor, celecoxib has different analgesic mechanisms to OxyContin and Pregabalin. Therefore, it could be beneficial to combine the administration of 3 kinds of drugs to exert their synergistic analgesic mechanism and reduce the amount of opioids and the possibility of adverse reactions.

Among the adverse reactions of opioids and pelvic and abdominal organ dysfunction caused by pudendal neuralgia, urinary dysfunction is the most troublesome, especially when complicated with urinary retention, which 
requires emergency treatment and even discontinuation of opioids, and affects the effective treatment of cancer pain. Tamsulosin hydrochloride sustained release capsules are a type of selective $\alpha 1$ adrenergic receptor blocker used to treat benign prostatic hyperplasia (BPH). Its main mechanism of action is to selectively block the $\alpha 1 \mathrm{~A}$ adrenergic receptor in the prostate. Relaxation of prostate smooth muscle (22) has the effect of improving symptoms such as dysuria caused by benign prostatic hyperplasia (23), which provides both theoretical and clinical bases for dysuria caused by opioids or pudendal nerve damage. The results of this study showed that the NRS score and the occurrence of urinary dysfunction in patients treated with celecoxib combined with OxyContin and Pregabalin were lower than those of patients treated with OxyContin combined with Pregabalin, indicating that celecoxib combined with OxyContin and Pregabalin treatment can effectively reduce the patient NRS scores and improve their quality of life. The combination of the 3 drugs played a certain complementary role in the analgesic mechanism, had a synergistic effect in the treatment of cancer pain, and to a certain extent reduced the problem of urinary dysfunction caused by the increase of opioids; due to the down-regulation of opioids compared to the control group. The dosage of similar drugs, when the same laxatives are prescribed, reduced the incidence of constipation, thereby improving digestive symptoms in patients with advanced tumors with pudendal neuralgia. This study suggests that the 3-drug combination therapy did not clearly increase drug-induced liver and kidney toxicity, except that the progress of the disease itself that affects liver and kidney function. For patients with urinary dysfunction receiving tamsulosin hydrochloride sustained-release capsule treatment, it has not been found to clearly increase the risk of drug-related liver and kidney toxicity, and as patients built tolerance to OxyContin (24), the symptoms of urinary dysfunction gradually subsided. Tamsulosin hydrochloride sustained-release capsules were stopped after 1 week of treatment, and all participants urinate smoothly. Tamsulosin hydrochloride sustainedrelease capsules may become an effective preventive drug for patients with cancerous pudendal neuralgia complicated with dysuria. Patients with advanced tumors have a limited survival period. It is more clinically meaningful to improve their quality of life through palliative treatment. This study compares the quality of life scores of patients at 24 hours, 1 week, 2 weeks, and 4 weeks after treatment. The 3 -drug combination can improve the quality of life of patients. There was no difference in the quality of life within 1 week, and the quality of life at 4 weeks between the 2 groups, but the quality of life was still improving compared with before treatment, and there was no significant adverse quality of life effect initiated by the 3 -drug combination treatment.

In summary, for cancer pain patients with pudendal neuralgia, especially patients with upregulation of opioid restriction due to dysuria, the application of celecoxib combined with OxyContin and Pregabalin has a significant short-term effect, can improve the patient's pain and reduce the occurrence of adverse reactions. Therefore, it is more practical in the real world to maximize the quality of life for patients with advanced tumors who are facing imminent death. This 3-drug combined analgesic method with the addition of tamsulosin hydrochloride sustained-release capsules when needed to prevent and treat urinary disorders is worthy of clinical promotion and application.

\section{Acknowledgments}

Funding: The work was supported by grants from Jilin Province Health and Health Technology Innovation Project (2020Z023).

\section{Footnote}

Reporting Checklist: The authors have completed the CONSORT reporting checklist. Available at http://dx.doi. org/10.21037/apm-21-590

Data Sharing Statement: Available at http://dx.doi. org/10.21037/apm-21-590

Conflicts of Interest: All authors have completed the ICMJE uniform disclosure form (available at http://dx.doi. org/10.21037/apm-21-590). The authors have no conflicts of interest to declare.

Ethical Statement: The authors are accountable for all aspects of the work in ensuring that questions related to the accuracy or integrity of any part of the work are appropriately investigated and resolved. All procedures performed in this study involving human participants were in accordance with the Declaration of Helsinki (as revised in 2013). This study was approved by Jilin Province People's Hospital ethics committee. All participants provided informed consent and signed an informed consent.

Open Access Statement: This is an Open Access article 
distributed in accordance with the Creative Commons Attribution-NonCommercial-NoDerivs 4.0 International License (CC BY-NC-ND 4.0), which permits the noncommercial replication and distribution of the article with the strict proviso that no changes or edits are made and the original work is properly cited (including links to both the formal publication through the relevant DOI and the license). See: https://creativecommons.org/licenses/by-nc-nd/4.0/.

\section{References}

1. van den Beuken-van Everdingen MH, Hochstenback LM, Joosten EA, et al. Update on prevalence of pain in patients with cancer: systematic review and meta-analysis. J Pain Symptom Manage 2016;51:1070-90.

2. Higginson IJ, Murtagh FE, Osborne TR. Epidemiology of pain in cancer. In: Hanna M, Zylicz Z, eds. Cancer Pain. London: Springer-Verlag; 2013:5-24.

3. Oosterling A, te Boveldt N, Verhagen C, et al. Neuropathic Pain Components in Patients with Cancer: Prevalence, Treatment, and Interference with Daily Activities. Pain Pract 2016;16:413-21.

4. Labat JJ, Riant T, Robert R, et al. Diagnostic criteria for pudendal neuralgia by pudendal nerve entrapment (Nantes criteria). Neurourol Urodyn 2008;27:306-10.

5. Dai J, Teng L, Zhao L, et al. The combined analgesic effect of pregabalin and morphine in the treatment of pancreatic cancer pain, a retrospective study. Cancer Med 2021;10:1738-44.

6. Zacny JP, Gutierrez S. Subjective, psychomotor, and physiological effects of oxycodone alone and in combination with ethanol in healthy volunteers. Psychopharmacology (Berl) 2011;218:471-81.

7. Zhou J, Wang Y, Jiang G. Oxycodone versus morphine for cancer pain titration: A systematic review and pharmacoeconomic evaluation. PLoS One 2020;15:e231763.

8. De Santis S, Borghesi C, Ricciardi S, et al. Analgesic effectiveness and tolerability of oral oxycodone/ naloxone and pregabalin in patients with lung cancer and neuropathic pain: an observational analysis. Onco Targets Ther2016;9:4043-52.

9. Fernandes K, Martins D, Juurlink D, et al. High-Dose Opioid Prescribing and Opioid-Related Hospitalization: A Population-Based Study. PLoS One 2016;11:e0167479.

10. Candy B, Jones L, Vickerstaff V, et al. Mu-opioid antagonists for opioid-induced bowel dysfunction in people with cancer and people receiving palliative care.
Cochrane Database Syst Rev 2018;6:CD006332.

11. Verhamme KMC, Sturkenboom MCJM, Stricker $\mathrm{BH}$, et al. Drug-induced urinary retention: incidence, management and prevention. Drug Saf 2008;31:373-88.

12. Hwang BY, Kwon JY, Lee DW, et al. A Randomized Clinical Trial of Nefopam versus Ketorolac Combined With Oxycodone in Patient-Controlled Analgesia after Gynecologic Surgery. Int J Med Sci 2015;12:644-9.

13. Garassino MC, Piva S, La Verde N, et al. Randomised phase II trial (NCT00637975) evaluating activity and toxicity of two different escalating strategies for pregabalin and oxycodone combination therapy for neuropathic pain in cancer patients. PLoS One 2013;8:e59981.

14. Ploteau S, Cardaillac C, Perrouin-Verbe MA, et al. Pudendal Neuralgia Due to Pudendal Nerve Entrapment: Warning Signs Observed in Two Cases and Review of the Literature. Pain Physician 2016;19:E449-54.

15. Elkins N, Hunt J, Scott KM. Neurogenic Pelvic Pain. Phys Med Rehabil Clin N Am 2017;28:551-69.

16. Gulyas M, Mattsson JSM, Lindgren A, et al. COX-2 expression and effects of celecoxib in addition to standard chemotherapy in advanced non-small cell lung cancer. Acta Oncol 2018;57:244-50.

17. Ibrahim MA, Abdelzaher WY, Rofaeil RR, et al. Efficacy and safety of combined low doses of either diclofenac or celecoxib with gabapentin versus their single high dose in treatment of neuropathic pain in rats. Biomed Pharmacother 2018;100:267-74.

18. Ji YB, Bo CL, Xue XJ, et al. Association of Inflammatory Cytokines With the Symptom Cluster of Pain, Fatigue, Depression, and Sleep Disturbance in Chinese Patients With Cancer. J Pain Symptom Manage 2017;54:843-52.

19. Li Z, Zeng J, Nie H, et al. Effectiveness of combined Pregabalin and Celecoxib for treatment of neuropathic pain after percutaneous endoscopic lumbar discectomy. Zhongguo Xiu Fu Chong Jian Wai Ke Za Zhi 2017;31:215-21.

20. Liu Z, Xu Y, Liu ZL, et al. Combined application of diclofenac and celecoxib with an opioid yields superior efficacy in metastatic bone cancer pain: a randomized controlled trial. Int J Clin Oncol 2017;22:980-5.

21. Strawson J. Nonsteroidal anti-inflammatory drugs and cancer pain. Curr Opin Support Palliat Care 2018;12:102-7.

22. Yoshida M, Inadome A, Masunaga K, et al. Effectiveness of tamsulosin hydrochloride and its mechanism in improving nocturia associated with lower urinary tract symptoms/ benign prostatic hyperplasia. Neurourol Urodyn 2010;29:1276-81. 
23. Karavitakis M, Kyriazis I, Omar MI, et al. Management of Urinary Retention in Patients with Benign Prostatic Obstruction: A Systematic Review and Meta-analysis. Eur Urol 2019;75:788-98.

24. Littlejohn C, Baldacchino A, Bannister J. Chronic

Cite this article as: Hou J, Lin Y, Fang Y, Li X, Li XN, Yang Y, Liu N, Jiang X, Yu Y, Zhou Y, Mi X, Ni Z, Lu X, Jin NY. Clinical efficacy evaluation and adverse reaction prevention by combined treatment with three drugs in cancerous pudendal neuralgia patients. Ann Palliat Med 2021;10(5):5754-5762. doi: 10.21037/apm-21-590 non-cancer pain and opioid dependence. J R Soc Med 2004;97:62-5.

(English Language Editor: J. Jones) 